\title{
40-Gb/s Systems on G.652 Fibers: Comparison Between Periodic and All-at-the-End Dispersion Compensation
}

\author{
Anna Pizzinat, Alessandro Schiffini, Francesco Alberti, Francesco Matera, \\ Armando N. Pinto, Member, IEEE, Member, OSA, and Paulo Almeida
}

\begin{abstract}
In the literature, two system solutions have been proposed to overcome high dispersion problems typical of G.652 fibers at high bit rates $(40 \mathrm{~Gb} / \mathrm{s})$ : they are periodic and all-at-the-end dispersion compensation. We carry out an exhaustive comparison between the two methods that, up to this moment, have been studied separately. In the first part, we introduce a simplified model on strong dispersion management (DM) with intrachannel four-waves mixing (IFWM) and intrachannel cross-phase modulation (IXPM). We then carry out extensive numerical simulations of a complete system in order to verify the results as a function of the input average power and of the input pulsewidth. Finally, we tackle a typical system aspect, i.e., the influence of nonlinear effects on dispersion compensating fibers (DCFs).
\end{abstract}

Index Terms-Nonlinear distortion, optical fiber communication, optical fiber dispersion.

\section{INTRODUCTION}

I $\mathrm{N}$ THE LITERATURE, there are several papers dealing with $40-\mathrm{Gb} / \mathrm{s}$ transmission on single-channel or on wavelengthdivision multiplexed (WDM) systems. They focus generally on dispersion-managed (DM) links with different kinds of maps on nonzero dispersion (ITU G.655) fibers.

However, a large part of the fibers deployed in existing systems are conventional single-mode fibers (ITU G.652), and it would be very important to improve the capacity of systems with such fibers. There are a few papers [1]-[4] that report numerical and experimental results on this kind of fibers, but there are still a number of open issues-not only on which is the best dispersion compensation method to be used, but also on the tolerances acceptable in these systems and on their behavior with respect to several nonlinear effects.

A preliminary analysis should deal with the choice of the signal format; however, it is recognized [3] that for terrestrial 40-Gb/s systems on G.652 fibers and long amplifier spacing

Manuscript received November 5, 2001; revised June 3, 2002.

A. Pizzinat is with the Department of Information Engineering, University of Padova, 35131 Padova, Italy (e-mail: annap@ wave.dei.unipd.it).

A. Schiffini and F. Alberti are with Pirelli Laboratories, 20126 Milan, Italy (e-mail: alessandro.schiffini@ pirelli.com).

F. Matera is with the Fondazione Ugo Bordoni, 00142 Rome, Italy (e-mail: mat@fub.it).

A. N. Pinto is with the Institute of Telecommunications, 3810-193 Aveiro, Portugal, and also with the University of Aveiro, 3810-193 Aveiro, Portugal (e-mail: anp@det.ua.pt).

P. Almeida is with the Institute of Telecommunications, 3810-193 Aveiro, Portugal.

Digital Object Identifier 10.1109/JLT.2002.802222
(>80 km), the return-to-zero (RZ) format guarantees the best performances. As a consequence, we focus our analysis on RZ systems, and we compare two of the compensation methods presented in literature: periodic compensation and all-at-the-end compensation. Periodic compensation has already been investigated [1]-[3], with the conclusion that the system's best performances can be achieved introducing a small prechirp at the beginning of the link. On the other side, the all-at-the-end compensation scheme has been introduced quite recently [4], [5] and is based on the transmission of very short RZ pulses that are rapidly dispersed and allowed to reduce the effects of self-phase modulation (SPM). Our aim is to carry out a complete comparison on these two methods, which, up to this moment, is lacking.

After the description of the system setup under examination, the comparison is divided in two parts: at first, we introduce a theory on intrachannel cross-phase modulation (IXPM) and intrachannel four-waves mixing (IFWM), then we show the results of extensive simulations to verify the previous theory and to give useful indications for the system design and experimental implementation.

As a further step, we analyze an important system aspect, i.e., the tolerance versus the input power of the dispersion compensating fibers (DCFs). In fact, in the first part of the paper, we simulate ideal dispersion compensation, and in the last part, we take in consideration the effective typical nonlinear coefficient of a conventional DCF.

Part of the numerical results to be reported in this paper has already been confirmed by experiments carried out in the framework of the European project IST/ATLAS (All optical Terabit per second LAmbda Shifted transmission).

\section{SySTEM SETUP}

In this section, we report a scheme of the transmission system and all the parameters included in the simulations. As already explained, we consider the transmission on ITU G.652 fibers with $\beta_{2}=-20 \mathrm{ps}^{2} / \mathrm{km}, \beta_{3}=-0.1 \mathrm{ps}^{3} / \mathrm{km}, \gamma=1.3 \mathrm{~W}^{-1}$ $\mathrm{km}^{-1}$, and $\alpha=0.25 \mathrm{~dB} / \mathrm{km}$.

In the first part, we realize complete chromatic dispersion compensation by means of ideal linear devices. We then introduce DCFs with $\beta_{2}=102 \mathrm{ps}^{2} / \mathrm{km}, \beta_{3}=0.24 \mathrm{ps}^{3} / \mathrm{km}, \gamma=6$ $\mathrm{W}^{-1} \mathrm{~km}^{-1}$, and $\alpha=0.5 \mathrm{~dB} / \mathrm{km}$.

Up to this moment, we have not taken into account polarization mode dispersion (PMD) effects, since all fibers we are 
(a)

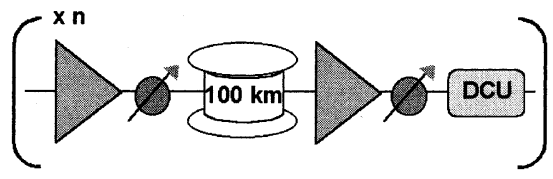

(b)

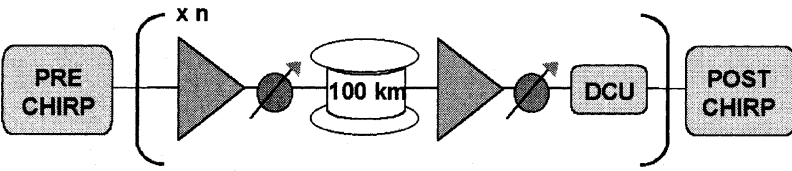

(c)

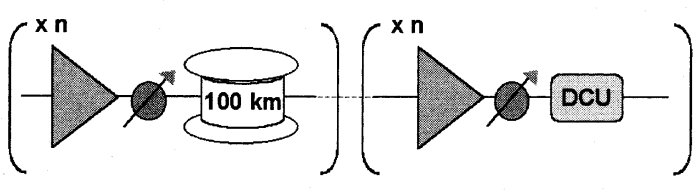

Fig. 1. Schematic of the link. DCU stands for dispersion compensation unit. Variable optical attenuators are placed after the amplifiers to change input power. (a) Periodic postcompensation scheme (dispersion management). (b) Periodic postcompensation with prechirp and postchirp. (c) All-at-the-end compensation (highly dispersive pulses).

going to use both in the laboratory and in the field experiment, for the ATLAS project, have an extremely low PMD coefficient $(0.04 \mathrm{ps} / \sqrt{\mathrm{km}})[6]$.

We simulate exact loss compensation by means of ideal erbium-doped fiber amplifiers (EDFAs) with inversion population factor $N_{\mathrm{sp}}=2$, and we do not introduce in-line filtering.

We obtain all the results by solving numerically the generalized Schrödinger equation with the split-step method [7], [8]. The interaction among pulses is investigated analyzing the propagation of pseudorandom sequences of different lengths (from 32 up to $512 \mathrm{~b}$ ).

The receiver is modeled as an ideal photodiode followed by a fourth-order Bessel-Thomson electrical filter with bandwidth $B=32 \mathrm{GHz}$. Then, the system performances are evaluated in terms of eye-opening penalty (EOP), defined as

$$
\mathrm{EOP}=-10 \log _{10}\left(\frac{\min \left({ }^{\prime} 1_{R}^{\prime}\right)-\max \left({ }^{\prime} 0_{R}^{\prime}\right)}{\min \left({ }^{\prime} 1_{\mathrm{btb}}^{\prime}\right)-\max \left({ }^{\prime} 0_{\mathrm{btb}}^{\prime}\right)}\right)
$$

where ${ }^{\prime} 1_{R}$ and ${ }^{\prime}{ }^{\prime}{ }_{R}$ are the amplitudes of the received marks and spaces, respectively, while ' 1 ' ${ }_{\text {btb }}$ and ' 0 ' btb are the amplitudes of marks and spaces in back-to-back.

Input pulses have a Gaussian shape with $T_{\mathrm{FWHM}}=5 \mathrm{ps}$, and average power between 0 and $+10 \mathrm{dBm}$. In this work, we focus our attention on a typical terrestrial link of $500 \mathrm{~km}$ with amplifiers' spacing of $100 \mathrm{~km}$.

Finally, we report the dispersion compensation schemes in Fig. 1. Fig. 1(a) refers to the periodic dispersion compensation, which can eventually be modified by adding an initial prechirp and a final postchirp, shown in Fig. 1(b). Fig. 1(c) corresponds to the all-at-the-end dispersion compensation scheme.

\section{TheORY ON PROPAGATION IN HIGHLY DisPERSIVE REGIME WITH IFWM AND IXPM}

Conventional single-mode fibers are characterized by a large amount of chromatic dispersion. Hence, optical pulses with a time width of a few picoseconds undergo a strong broadening and consequent pulses overlapping (e.g., $T_{\mathrm{FWHM}}=5 \mathrm{ps}$ corresponds to a dispersion length of $450 \mathrm{~m}$, and in about $4 \mathrm{~km}$, adja- cent pulses overlap completely, while the nonlinear length is of the order of hundreds of kilometers). This propagation regime has already been analyzed in literature and is usually divided in two branches: dispersion management (DM), which is based on periodic dispersion compensation [3], [9]-[11], and propagation of highly dispersive pulses (HDP) [4], [5], which allow the dispersion compensation all at the end of the system.

Since the dispersion length is much shorter than the nonlinear length, the nonlinearity can be considered as a small perturbation compared with the pulse distortion caused by dispersion. Hence, according to [9] through [11], the nonlinear Schrödinger (NLS) equation can be solved, assuming a solution in the form $U_{0}+\Delta U$, where $U_{0}$ is the linear solution and $\Delta U$ is the small nonlinear induced perturbation. Solving the modified NLS equation under these conditions, we obtain the following linear equation for $\Delta U$ :

$$
\frac{\partial \Delta U}{\partial z}+i \frac{\beta_{2}}{2} \frac{\partial^{2} \Delta U}{\partial t^{2}}+\frac{\alpha}{2} \Delta U=i \gamma\left|U_{0}\right|^{2} U_{0}
$$

Considering the propagation of two adjacent Gaussian pulses $U_{1}$ and $U_{2}$, and replacing $U_{0}$ by $U_{1}+U_{2}$, we obtain

$$
\begin{aligned}
\frac{\partial \Delta U}{\partial z}+i \frac{\beta_{2}}{2} \frac{\partial^{2} \Delta U}{\partial t^{2}}+\frac{\alpha}{2} \Delta U & \{\underbrace{}_{\mathrm{IXPM}}=i \gamma\{\underbrace{\left|U_{1}\right|^{2} U_{1}+\left|U_{2}\right|^{2} U_{2}}_{\mathrm{SPM}} \\
& +\underbrace{2\left|U_{2}\right|^{2} U_{1}+2\left|U_{1}\right|^{2} U_{2}}_{\mathrm{IFWM}} \\
& +\underbrace{U_{1} U_{2}^{*} U_{1}+U_{2} U_{1}^{*} U_{2}}_{\text {UXM }}\} .
\end{aligned}
$$

The first couple of terms on the right-hand side (RHS) of (2) is due to SPM, the second one is responsible for the IXPM, and the last one is the cause of the IFWM. These three nonlinear effects induce pulse distortion, timing jitter, and energy fluctuations, respectively. For transmission distances of few hundreds of kilometers, the pulse distortion induced by the SPM is a minor effect. On the other hand, the timing jitter and energy fluctuations induced by the IXPM and the IFWM, respectively, can assume a major role in the system performance.

Considering just the IXPM, each pulse suffers an instantaneous frequency deviation due to the presence of the neighboring one. As found in [9], the leading pulse $U_{1}$ suffers an instantaneous frequency deviation in an infinitesimal distance $d z$ due to the presence of $U_{2}$ and it reads

$$
\omega_{1}=-2 \gamma d z \frac{d\left|U_{2}\right|^{2}}{d t} \text {. }
$$

This instantaneous frequency deviation induces a pulse central frequency shift $\delta \omega$, which after propagation over a distance $L$, produces a timing shift given by $\delta t=L \beta_{2} \delta \omega$.

The central frequency shift of the leading pulse evolves according with the curves presented in Fig. 2. It should be noted that IXPM produces a pulse red shift, which is less critical in the HDP scheme. In fact, after the first span, the central frequency shift is completely different in the two dispersion compensa- 


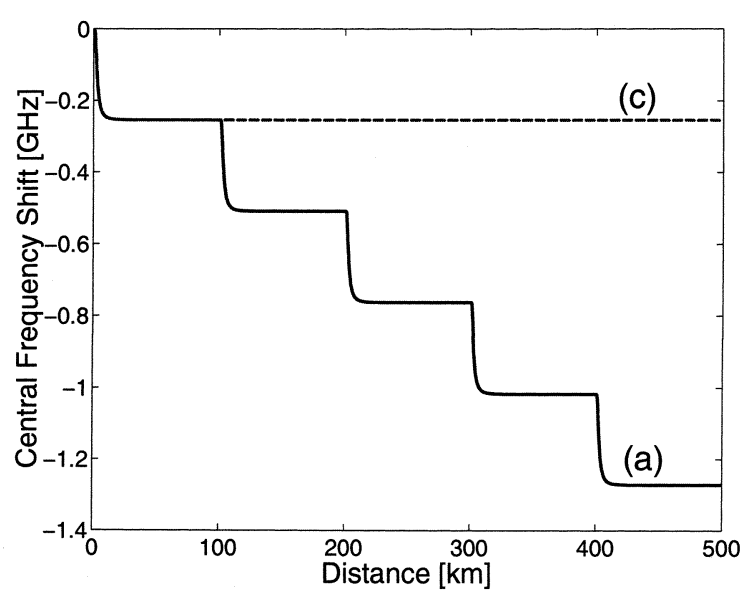

Fig. 2. Pulse central frequency deviation for optical input average power $P_{\text {in }}=8 \mathrm{dBm}$. Line (a) corresponds to DM scheme. Line (c) corresponds to all-at-the-end compensation.

tion schemes. In the DM case [line (a)], the pulse recovers its initial shape after the chromatic dispersion compensation device, giving rise to a similar behavior in all the spans. In the HDP scheme [line (c)], the leading pulse central frequency shift due to the pulse-to-pulse interaction becomes very small after a few kilometers of propagation, despite the huge overlap between pulses. This happens because the pulses broaden continuously until the end of the link, which reduces the pulses derivative and changes its sign across the overlap region. Actually, this confirms once more the theory reported in [9] and [10].

Regarding IFWM, it should be noted that the last two terms in (2) allow power to flow from the signal pulse slots to adjacent bit slots, giving rise to the so-called "shadow" or "ghost" pulses that grow in bit slots carrying zeros or to amplitude fluctuations in bit slots carrying ones [5], [9]-[11]. Using (2), we derive the expression for the perturbation induced by the IFWM terms in the frequency domain, as follows:

$$
\begin{aligned}
\Delta \hat{U}(z, \omega)=\exp & \left(-\frac{\alpha}{2} z+i \frac{\beta_{2}}{2} \omega^{2} z\right) \\
& \times \int_{0}^{z} \hat{f}(\zeta, \omega) \exp \left(+\frac{\alpha}{2} \zeta-i \frac{\beta_{2}}{2} \omega^{2} \zeta\right) d \zeta
\end{aligned}
$$

where $\hat{f}(\zeta, \omega)$ is the Fourier transform of the IFWM terms, shown in (2). From (4), we obtain the energy transferred by the IFWM process, as follows:

$$
E_{\mathrm{IFWM}}=\frac{1}{2 \pi} \int_{-\infty}^{+\infty} \Delta \hat{U} \Delta \hat{U}^{*} d \omega .
$$

Fig. 3 shows the percentage of energy in the "shadow" pulses due to the $U_{1} U_{2}^{*} U_{1}$ IFWM term [see (2)]. According to [5], we plotted the ratio between the mean energy in the "shadow" pulses and the mean energy in the marks. The IFWM process gives rise to a closure in the eye vertical aperture and is much more efficient in the DM case [line (a)] than in the HDP scheme [line (c)] in the assumption of two adjacent pulses. In fact, in the DM case, the perturbation field originated by the IFWM is enhanced by an identical contribution in all spans, because of the

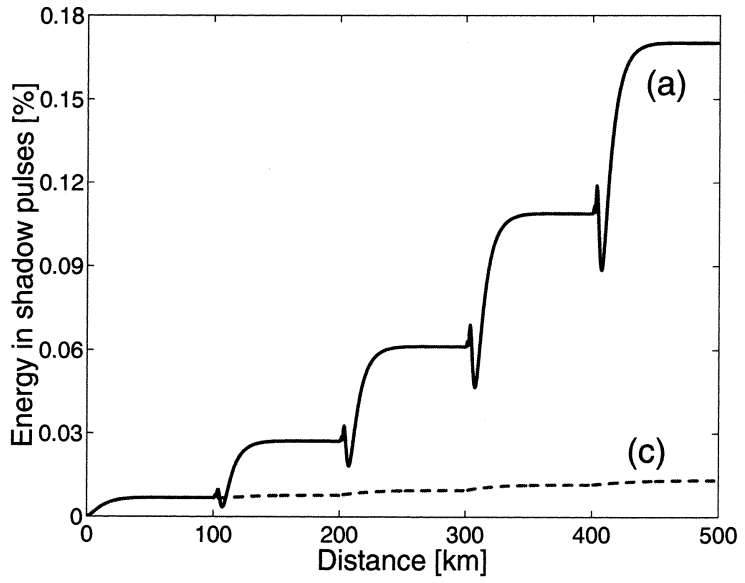

Fig. 3. Percentage of energy in "shadow" pulses for $P_{\text {in }}=8 \mathrm{dBm}$. Line (a) corresponds to DM scheme. Line (c) corresponds to all-at-the-end compensation.

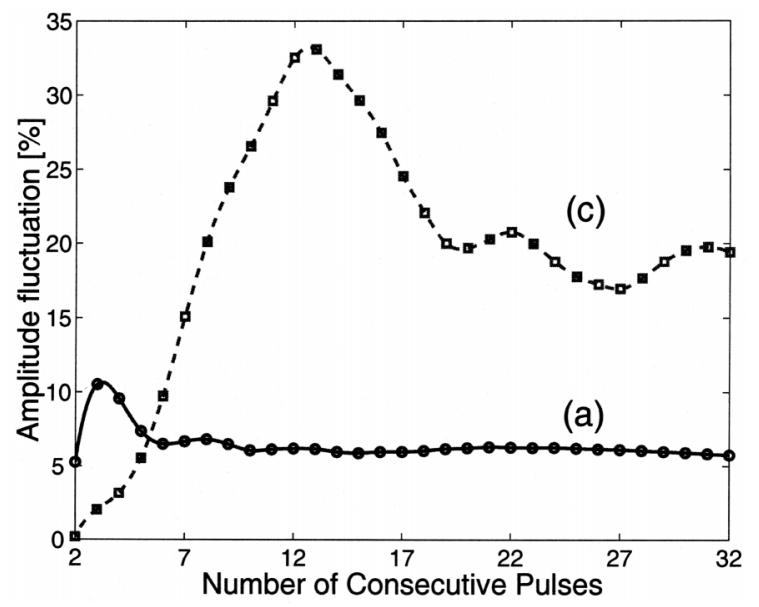

Fig. 4. Amplitude fluctuation on the marks as function of the number of consecutive pulses, after $5 \times 100 \mathrm{~km}$ of G.652 fiber. The amplitude fluctuation is calculated as the ratio between the standard deviation of the peak pulse power and the mean peak power of ones [5]. Line (a) corresponds to DM scheme. Line (c) corresponds to all-at-the-end compensation.

periodicity of the dispersion map. As a consequence, the energy in "shadow" pulses increases according to a quadratic law with the number of spans. This can be seen clearly in Fig. 3, and confirms the result predicted in [9] and [10].

However, a radical change occurs when long sequences of pulses are considered. Fig. 4 shows the pulses' peak power standard deviation, measured at the end of the system, for sequences of consecutive marks of various lengths. Indeed, for more than five consecutive pulses, the HDP system presents a larger fluctuation in the marks compared with the DM system. This can be understood by comparing the pulse breath dynamics in the two schemes. In the DM scheme, the pulses recover their initial width at the end of each span so that the pulse-to-pulse nonlinear interaction is limited to the neighboring pulses. On the contrary, the pulsewidth in the HDP scheme increases proportionally to the propagation distance and so does the number of interacting pulses. This effect produces, in turn, a considerable increase in the pulses' amplitude fluctuation up to approximately 15 consecutive marks. After this point, it can be observed in Fig. 4 that 

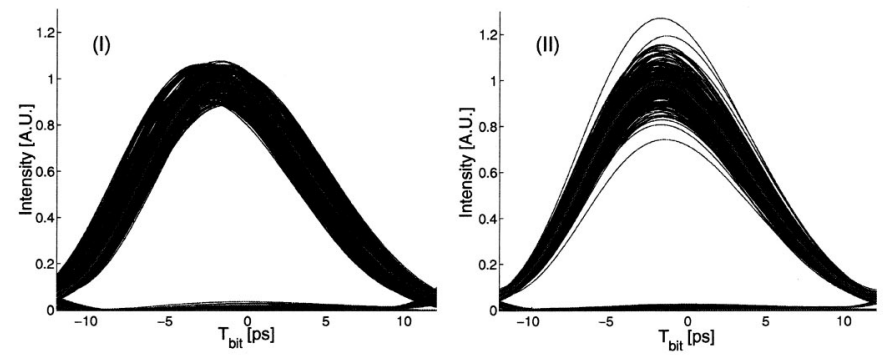

Fig. 5. Eye diagrams after $500 \mathrm{~km}$ of propagation. (I): Periodic postcompensation. (II) All-at-the-end compensation. Simulations were carried out with parameters described in Section II for a pseudorandom sequence of $512 \mathrm{~b}$ and input average power of $8 \mathrm{dBm}$.

the amplitude fluctuation remains high and presents a behavior similar to the DM regime, which means that it is almost independent of the number of consecutive pulses. In real systems, random pulse sequences cross the system, and, consequently, this long-range nonlinear interaction is the cause of a large eye penalty in the HDP scheme.

In accordance with these results, we expect that the maximum achievable distance will be determined by the timing jitter in the DM scheme and by the amplitude fluctuation in the HDP scheme.

\section{Simulation Results}

The aim of this section is to verify the previous results with extensive simulations, according to the parameters introduced in Section II.

Fig. 5 reports the eye diagrams corresponding to the system of $500 \mathrm{~km}$ for a pseudorandom sequence of $512 \mathrm{~b}$ and input average power $+8 \mathrm{dBm}$. The eye diagram on the left-hand side corresponds to periodic postcompensation and is clearly affected by timing jitter. The eye diagram on the RHS corresponds to all-at-the-end dispersion compensation; while the timing jitter is negligible, there is a visible amplitude fluctuation. Moreover, the energy in "shadow" pulses is negligible for this system length, pulsewidth, and peak power. In order to calculate exactly nonlinear intrachannel effects in the two schemes, we evaluate the timing jitter as the standard deviation of the pulse position, as follows:

$$
\operatorname{std}\left(\frac{\int t|U(L, t)|^{2} d t}{\int|U(L, t)|^{2} d t}\right)
$$

where $L$ is the propagation distance. The amplitude fluctuation is the ratio between the standard deviation of the pulse energy and the mean energy of ones [5]. Figs. 6 and 7 report these two quantities as a function of the propagation distance for the DM scheme [line (a)] and the HDP one [line (c)], confirming the results shown in Figs. 2 and 4.

At this point, we would like to understand whether the overall system is affected more strongly by the DM timing jitter or by the HDP amplitude fluctuation. We made simulations in the following cases: postcompensation, prechirp + postcompensation + postchirp and all-at-the-end compensation (a simple scheme of the three cases is shown in Fig. 1). Fig. 8 summarizes the results reporting the EOP versus the input average power. It can be noted that the three curves have the same behavior for low

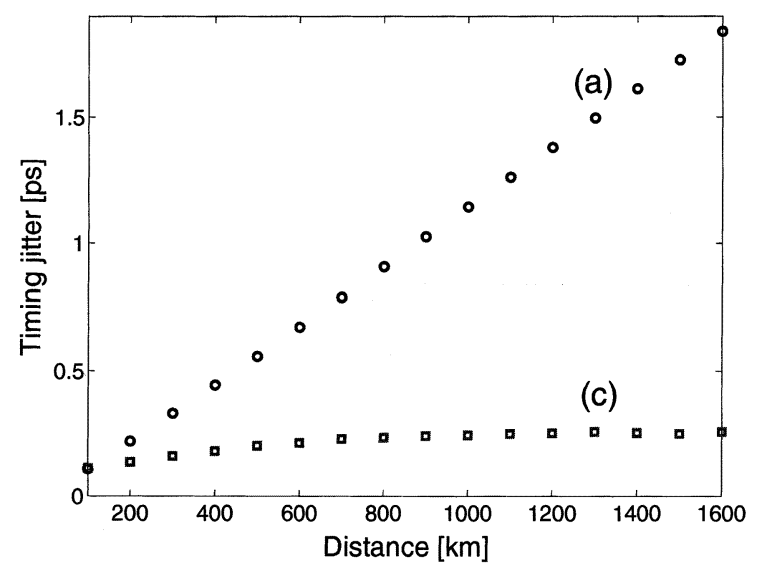

Fig. 6. Timing jitter versus propagation distance. Line (a) corresponds to periodic dispersion compensation (DM). Line (c) corresponds to all-at-the-end compensation (HDP). Simulations were carried out in the same conditions as in Fig. 5.

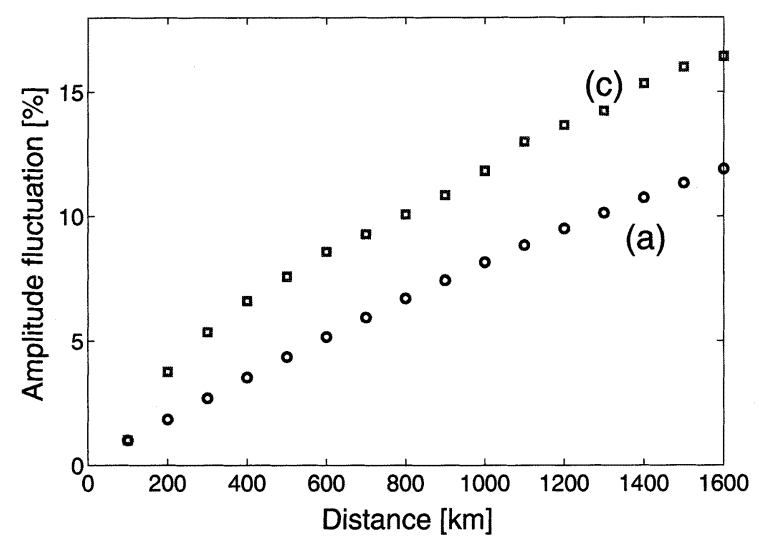

Fig. 7. Amplitude fluctuation versus propagation distance. Line (a) corresponds to periodic dispersion compensation (DM). Line (c) corresponds to all-at-the-end compensation (HDP). Simulations were carried out in the same conditions as in Fig. 5.

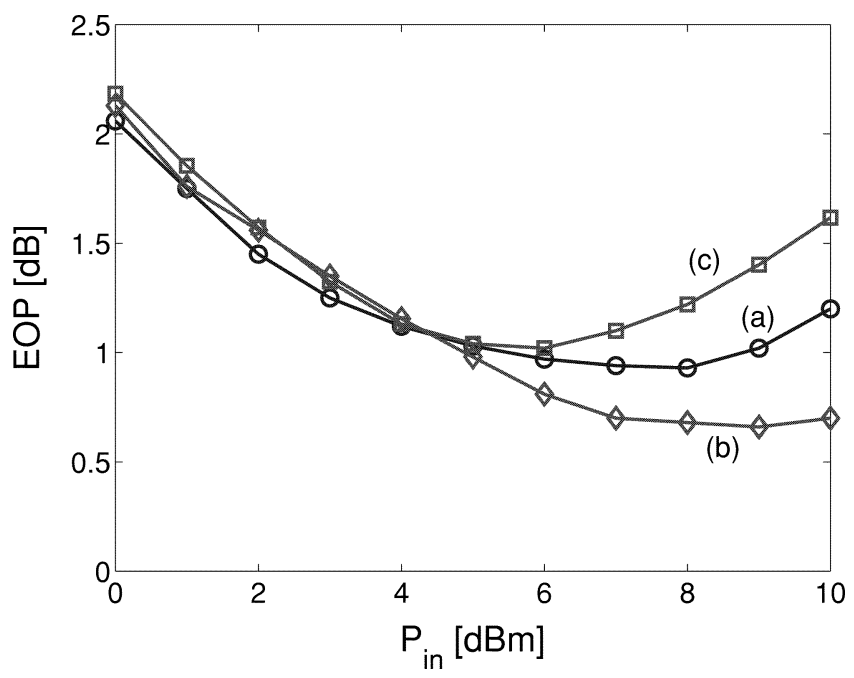

Fig. 8. EOP versus input average power $\left(P_{\text {in }}\right)$ for different schemes. Line (a): DM. Line (b): Prechirp $100 \mathrm{ps}^{2}+$ DM. Line (c): All-at-the-end compensation.

input powers, when the signal-to-noise ratio (SNR) is small. For larger values of input power, the nonlinear effects prevail over 



Fig. 9. EOP versus time width at half-maximum of the transmitted pulses. (I) corresponds to input average power of $5 \mathrm{dBm}$. (II) corresponds to input average power of $10 \mathrm{dBm}$. Line (a): DM. Line (b): DM with optimum prechirp. Line (c): HDP.

any other source of distortion, but their influence is different for the three compensation schemes. The postcompensation scheme [line (a)] is affected by SPM for average powers larger than 7 $\mathrm{dBm}$. On the other side, the optimum choice for the prechirp [line (b)] in Fig. 8 guarantees the same EOP of an ideal linear system [3], [12], up to $8 \mathrm{dBm}$. By means of simulations, we obtained an optimum prechirp of $100 \mathrm{ps}^{2}$ that agrees with the one obtained theoretically in [3]. In the all-at-the-end compensation scheme [line (c)], the system performances present an optimum for an input power of $6 \mathrm{dBm}$. For higher input powers, the EOP becomes worse than the one with optimum prechirp. As a consequence, in a 500-km-long link in the HDP scheme, the EOP curve's behavior should not be attributed to timing jitter but to amplitude fluctuations.

In order to complete the comparison between DM with or without optimum prechirp and HDP, we fix the input power and vary the pulse time width. Fig. 9 reports the EOP for two different input powers and three compensation schemes: DM [line (a)], DM with optimum prechirp [line (b)], and HDP [line (c)].

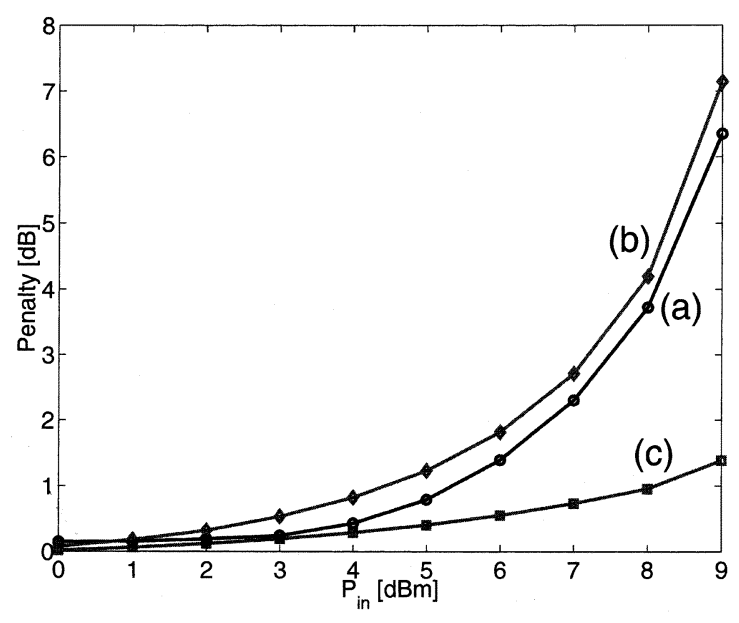

Fig. 10. Penalty versus input average power in DCF. The penalty has been calculated as the difference between the eye opening of the system with ideal DCF and the one with real DCF (which includes also nonlinear effects). Line (a): DM. Line (b): DM with optimum prechirp. Line (c): HDP scheme.

It can be noted that the DM with optimum prechirp guarantees better results also for different pulsewidths for all input powers, as already observed in Fig. 8. While the differences between the three curves are negligible for $P_{\text {in }}=5 \mathrm{dBm}$ [Fig. 9(I)], the advantage of the optimum prechirp becomes consistent for increasing values of the input power [Fig. 9(II)].

Another important comment deals with the optimum pulsewidth: in literature [4], [5], the HDP scheme was adopted only with pulses as short as 3 ps. In contrast, Fig. 9 shows that it is possible to obtain fine results also with larger pulses that are certainly easier to be generated in practice. In conclusion, longer pulses and HDP scheme, in spite of a small performance degradation, allow quite good results with a much simpler system.

\section{System AsPeCts: NonlineAR EFFECTS IN DCF}

Up to this point, we supposed that we would be able to achieve exact chromatic compensation and to have ideal dispersion compensation devices. It is known that in a $40-\mathrm{Gb} / \mathrm{s}$ system, the tolerance for chromatic dispersion compensation is a critical issue when considering G.652 fibers. In particular, we verified that for the system described in Section II, an EOP of $1 \mathrm{~dB}$ corresponds to a difference on the span length of only $1 \mathrm{~km}$. On the other side, though several new methods, namely Bragg gratings and higher order modes, for fixed and tunable dispersion compensation are the object of research, most of the installed systems deploy fibers for dispersion compensation [13]. A commercially available DCF module allows compensation only for a fixed value of dispersion, and consequently, the postcompensation scheme with optimum prechirp becomes practically unfeasible.

Moreover, two other drawbacks of DCF are the high losses that force the adoption of double-stage amplifiers and the small effective area that leads to a large nonlinear coefficient. This means that the DCF input power is actually a critical parameter and should be considered in the design of an optical transmission system. 
Fig. 10 shows the penalty versus the average optical input power in the DCF. The simulations were done considering an amplifier before the DCF, as shown in Fig. 1; amplified spontaneous emission (ASE) noise has not been included in order to focus the attention on nonlinear effects in the DCF. As usual, we made simulations for the following dispersion compensation schemes: DM [line (a)], DM with optimum prechirp [line (b)], and HDP [line (c)]. Our purpose is to show the tolerance of the three kinds of compensation toward DCF nonlinearity, and consequently, we calculated the penalty as the difference between the eye opening of the system with ideal DCF $(\gamma=0)$ and the one with real nonlinear DCF $\left(\gamma=6 \mathrm{~W}^{-1} \mathrm{~km}^{-1}\right)$. In this way, it can be demonstrated that the periodic behavior described in [3] for the DM with optimum prechirp is no longer valid; in addition, the HDP scheme is more robust with respect to these effects.

\section{CONCLUSION}

In this paper, we reported a comparison between DM and HDP dispersion compensation schemes on G.652 fibers. Such fibers are characterized by high chromatic dispersion, which makes it quite difficult to realize $40-\mathrm{Gb} / \mathrm{s}$ systems with long amplifiers spacing.

In conditions of perfect compensation and ideal dispersion compensating devices, the DM scheme with optimum prechirp guarantees the best performances in terms of bit error rate (BER), in spite of a larger timing jitter. The HDP scheme allows a BER quite similar to the one of the DM scheme for a good range of input pulsewidths. Moreover, if one considers real drawbacks typical of long-haul terrestrial systems, such as non-exact dispersion compensation and nonlinear effects in the DCF, the result is that the HDP scheme is the only one that achieves a good compromise between performances, feasibility, and simplicity.

\section{ACKNOWLEDGMENT}

This work was carried out in the framework of the IST/ATLAS project. The authors would like to thank A. Galtarossa, P. Franco, and J. Ferreira da Rocha for helpful discussions and suggestions.

\section{REFERENCES}

[1] C. M. Weinert, R. Ludwig, W. Pieper, H. G. Weber, D. Breuer, K. Petermann, and F. Kuppers, " $40 \mathrm{~Gb} / \mathrm{s}$ and $4 \times 40 \mathrm{~Gb} / \mathrm{s}$ TDM/WDM standard fiber transmission," J. Lightwave Technol., vol. 17, pp. 2276-2283, Nov. 1999.

[2] A. Sano, Y. Miyamoto, S. Kuwahara, and H. Toba, "A 40 Gb/s/ch transmission with SPM/XPM suppression through prechirping and dispersion management," J. Lightwave Technol., vol. 18, pp. 1519-1527, Nov. 2000.

[3] M. Zitelli, F. Matera, and M. Settembre, "Single-channel transmission in dispersion management links in conditions of very strong pulse broadening: Application to $40 \mathrm{~Gb} / \mathrm{s}$ signals on step-index fibers," J. Lightwave Technol., vol. 17, pp. 2498-2505, Dec. 1999

[4] S. G. Park, A. H. Gnauck, J. M. Wiesenfeld, and L. D. Garrett, " $40 \mathrm{~Gb} / \mathrm{s}$ transmission over multiple $120 \mathrm{~km}$ spans of conventional single-mode fiber using highly dispersed pulses," IEEE Photon. Technol. Lett., vol. 12, pp. 1085-1087, Aug. 2000.
[5] A. Mecozzi, C. Balslev Clausen, and M. Shtaif, "System impact of intrachannel nonlinear effects in highly dispersed optical pulse transmission," IEEE Photon. Technol. Lett., vol. 12, pp. 1633-1635, Dec. 2000.

[6] A. Galtarossa, L. Palmieri, A. Pizzinat, M. Schiano, and T. Tambosso, "Measurement of local beat length and differential group delay in installed single-mode fibers," J. Lightwave Technol., vol. 18, pp. 1389-1394, Oct. 2000.

[7] G. P. Agrawal, Nonlinear Fiber Optics. San Diego, CA: Academic, 1995.

[8] E. Iannone, F. Matera, A. Mecozzi, and M. Settembre, Nonlinear Optical Communication Networks. New York: Wiley, 1998.

[9] P. V. Mamyshev and N. A. Mamysheva, "Pulse-overlapped dispersionmanaged data transmission and intrachannel four wave mixing," Opt. Lett., vol. 24, pp. 1454-1456, 1999.

[10] M. J. Ablowitz and T. Hirooka, "Resonant nonlinear intrachannel interactions in strongly dispersion-managed transmission systems," Opt. Lett., vol. 25, pp. 1750-1752, 2000.

[11] P. Jhanisson, D. Anderson, A. Berntson, and J. Martensson, "Generation and dynamics of ghost pulses in strongly dispersion-managed fiber-optic communication systems," Opt. Lett., vol. 26, pp. 1227-1229, 2001.

[12] C. Peucheret, N. Hanik, R. Freund, L. Molle, and P. Jeppesen, "Optimization of Pre- and Post-dispersion compensation schemes for 10-Gbit/s NRZ links using standard and dispersion compensating fibers," IEEE Photon. Technol. Lett., vol. 12, pp. 992-994, Aug. 2000.

[13] V. Srikant, "Broadband dispersion and dispersion slope compensation in high bit rate and ultra long haul systems," presented at the Tech. Dig. Optical Fiber Conf. 2, Anaheim, CA, 2001. TuH1.

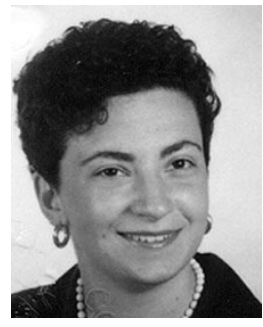

Anna Pizzinat was born in Treviso, Italy, in 1974. She received the Master degree in electronic engineering from the University of Padova, Padova, Italy, in 1999. Currently, she is pursuing the Ph.D. degree at the same university.

In 2002, she spent six months at the University of Maryland Baltimore County, Baltimore, MD, as a visiting student. Her research interests include the study of fibers with low polarization mode dispersion and the analysis and design of high-bit-rate optical transmission systems.

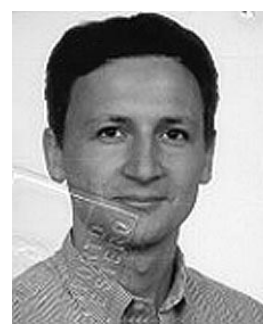

Alessandro Schiffini was born in Ivrea, Italy, in 1970. He received the physics degree from the University of Torino, Turin, Italy, in 1995. From 1994 to 1997, he attended the Cselt Laboratories, Turin, Italy, where he studied soliton mode-locked fiber lasers.

In 1997, he joined Pirelli Cavi e Sistemi, working in the Pirelli advanced photonics research group on high-speed transmission systems. In particular, his main areas of activity involve theory, design, and experimental investigation on $40-80 \mathrm{~Gb} / \mathrm{s}$ telecommunications systems. He has authored and coauthored more than 20 scientific papers in international journals and conferences. Since 2001, he has been with the WDM System Technology Lab of Pirelli Labs Optical Innovation.

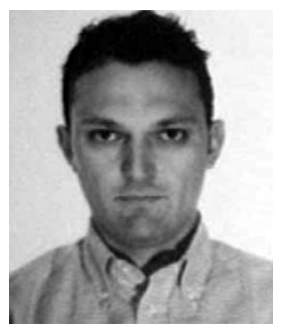

Francesco Alberti was born in Saronno, Italy, in 1969. He received the degree in electronic engineering from the Politecnico of Milan, Milan, Italy, in 2000.

In 1998, he worked at Instrumentation Devices in the field of measurements for automotive and avionics applications. In 1999, he joined the Speciality Fibres Group of Pirelli Cavi e Sistemi, studying an all-optical switch based on resonant nonlinear effects in doped fibers. Since 2000, he has been with Pirelli Advanced Photonics Research, working on theoretical and experimental investigations of high-speed $(40-80 \mathrm{~Gb} / \mathrm{s})$ telecommunications systems. Since 2001, he has been with the WDM System Technology Lab of Pirelli Laboratories Optical Innovation. 
Francesco Matera, photograph and biography not available at time of publication.

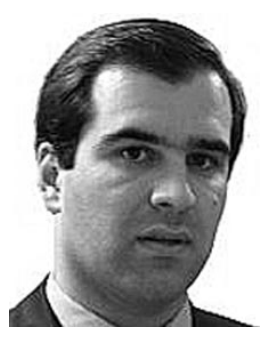

Armando N. Pinto (M’00) received the B.S. degree in electronic and telecommunications engineering and the Ph.D. degree in electrical engineering from Aveiro University in 1994 and 1999, respectively.

He joined the Institute of Telecommunications in 1995 and the Electronic and Telecommunications Department of Aveiro University in October 1997 Currently, he is Assistant Professor in the Electronic and Telecommunications Department of Aveiro University, where he has been teaching telecommunications, computer science and computer networks courses. He is also a Senior Researcher at the Institute of Telecommunications. His main research interests focus on optical communication systems, nonlinear transmission, multiwavelength optical communication systems and optical networks.

Dr. Pinto is a Member of the Lasers \& Electro-Optics Society (LEOS), the Communication Society (COMSOC), and the Optical Society of America (OSA).
Paulo Almeida, photograph and biography not available at time of publication. 